\title{
THE LEAST $r$-FREE NUMBER IN AN ARITHMETIC PROGRESSION
}

BY

\author{
KEVIN S. MCCURLEY
}

\begin{abstract}
Let $n_{r}(a, q)$ be the least $r$-free number in the arithmetic progession $a$ modulo $q$. Several results are proved that give lower bounds for $n_{r}(a, q)$, improving on previous results due to Erdös and Warlimont. In addition, a heuristic argument is given, leading to two conjectures that would imply that the results of the paper are close to best possible.
\end{abstract}

1. Introduction. If $r$ is at least 2, define $n_{r}(a, q)$ as the least positive integer in the arithmetic progression $a$ modulo $q$ that is not divisible by the $r$ th power of a prime, and define

$$
n_{r}^{*}(q)=\max _{(a, q)=1} n_{r}(a, q), \quad n_{r}(q)=\max _{(a, q) r \text {-free }} n_{r}(a, q) .
$$

In this paper we give some lower bounds for these functions, and state some conjectures concerning their rate of growth.

There are several known upper bound results. Prachar $[8]$ has shown that

$$
n_{r}^{*}(q) \ll q^{1+1 / r} \exp \left(\frac{r}{r-1} \omega(q) \log r\right),
$$

where $\omega(q)$ is the number of distinct prime factors of $q$. If $r$ is large as a function of $q$, the work of Cohen and Robinson [1] yields the sharper estimates

$$
n_{r}^{*}(q) \ll q^{1+1 /(r-1)}, \quad n_{r}(q) \ll\left(q^{2} / \varphi(q)\right)^{1+1 /(r-1)} .
$$

Furthermore any result is trivial if $r$ exceeds $\log q / \log 2$, since $n_{r}(a, q)$ does not exceed $q$ in this case.

In the case when $r$ is 2, improvements in Prachar's upper bound result have been made by Erdös [2] and more recently by Heath-Brown [3], who proved that

$$
n_{2}(q) \ll q^{13 / 9}(d(q) \log q)^{6},
$$

where $d(q)$ is the number of divisors of $q$. Hooley [4] has also shown that $n_{2}^{*}(q) \ll q^{4 / 3+\varepsilon}$ for a sequence of $q$ 's having positive lower density. Further results concerning averages of $n_{2}(a, q)$ have been given by Warlimont [13].

As for lower bounds, Warlimont [12] proved that for every $C \geqslant 1$ there exists an $\varepsilon=\varepsilon(C)$ such that $n_{2}(a, q)$ exceeds $C q$ for infinitely many $q$ and at least $\varepsilon \varphi(q)$ values of $a$ for each $q$. Erdös [2] stated without proof that

$$
n_{2}^{*}(q) \neq o\left(q \frac{\log q}{\log \log q}\right)
$$

Received by the editors November 16,1983. The contents of this paper were presented on March 18 , 1983 at the 802nd Meeting of the American Mathematical Society in Norman, Oklahoma.

1980 Mathematics Subject Classification. Primary 10H15, 10H20.

(C1986 American Mathematical Society $0002-9947 / 86 \$ 1.00+\$ .25$ per page 
and Warlimont [12] gave a proof that there exist infinitely many $q$ with

$$
n_{2}^{*}(q)>\left(\frac{1}{3}-\varepsilon\right) q \frac{\log q}{\log \log q}
$$

The values of $q$ constructed by Warlimont were quite rare, being a product of many small prime factors, and one might be led to believe that (1) occurs only very rarely. In fact, we show that a slightly stronger result is true for all $q$, and for a large number of residue classes for each $q$.

THEOREM 1. If $\varepsilon>0$ and $\log q /(r \log \log q)$ is sufficiently large, then there exist at least

$$
\exp \left(\frac{1-\varepsilon}{r}\left(1-\frac{\log r}{\log \log q}\right) \log q\right)
$$

values of $a$ with $(a, q)=1,0<a<q$, and

$$
n_{r}(a, q)>\frac{1-\varepsilon}{r} q \frac{\log q}{\log \log q} .
$$

For some values of $q$ the size of the constant $(1-\varepsilon) / r$ in (2) can be improved slightly, but for this we pay a price in the number of residue classes $a$ for which the estimate is known to hold.

THEOREM 2. If $\varepsilon>0, \log q /(r \log \log q)$ is sufficiently large, and $q$ is not divisible by the first $[\log q /(r \log \log q)]$ primes, then

$$
n_{r}^{*}(q)>(1-\varepsilon) \frac{\zeta(r)}{r} q \frac{\log q}{\log \log q} .
$$

If we focus our attention on a particular value of $a$, then the following result gives positive information.

THEOREM 3. Let $r$ be fixed, $\varepsilon>0$, and $a>0$. If $a$ is not $r$-free, then there exist infinitely many $q$ with $(a, q)=1$ and

$$
n_{r}(a, q)>\frac{1-\varepsilon}{r} q \frac{\log q}{\log \log q} .
$$

The previous results have been concerned with the residue classes $a$ with $(a, q)=1$. If we relax this condition, then we can obtain the following improvement.

THEOREM 4. For each $r$ and $\varepsilon>0$ there exist infinitely many $q$ such that

$$
n_{r}(q)>\frac{e^{\gamma}-\varepsilon}{r} q \log q \log _{2} q \frac{\log _{4} q}{\left(\log _{3} q\right)^{2}},
$$

where $\log _{n} q$ is the $n$-fold iterated logarithm and $\gamma$ is Euler's constant.

This result is very similar to the best known lower bounds of Prachar [9] and Pomerance [7] for the least prime in an arithmetic progression.

There may still be improvements that can be made here, particularly in the distribution of $n_{r}(a, q)$ about its mean value. 
2. Some conjectures. There is still a large gap between the lower bounds presented here and the upper bound results of Prachar and Heath-Brown. In this section we give heuristic arguments for two conjectures concerning the order of magnitude of $n_{r}^{*}(q)$ and $n_{r}(q)$. For simplicity we shall assume that $r$ is fixed in this section.

Previously Erdös [2] conjectured that $n_{r}^{*}(q) \ll q^{1+\varepsilon}$, and to this we add the following conjectures.

CONJECTURE 1 . Let $C_{r}(q)=\prod_{p+q}\left(1-p^{-r}\right)$. Then

$$
\varlimsup_{q \rightarrow \infty} \frac{n_{r}^{*}(q)}{q \log q /-\log \left(1-C_{r}(q)\right)}=2 .
$$

Furthermore there exists a sequence $S$ with asymptotic density zero such that

$$
\lim _{\substack{q \rightarrow \infty \\ q \notin S}} \frac{n_{r}^{*}(q)}{q \log q /-\log \left(1-C_{r}(q)\right)}=1 .
$$

CONJECTURE 2. Let

$$
D_{r}(q)=\prod_{p^{r}+q}\left(1-\left(p^{r}, q\right) p^{-r}\right) .
$$

If $\varepsilon>0$, then for $q$ sufficiently large we have

$$
n_{r}(q)<(2+\varepsilon) q \frac{\log q}{-\log \left(1-D_{r}(q)\right)} .
$$

It is interesting to compare the conjectures with the results stated in $\S 1$. If $q$ is the product of all primes not exceeding $z$, with $z$ large, then

$$
\begin{aligned}
C_{r}(q) & =\exp \left(\sum_{p>z} \log \left(1-p^{-r}\right)\right)=\exp \left(-\sum_{p>z} p^{-r}+O\left(z^{1-2 r}\right)\right) \\
& =1-\sum_{p>z} p^{-r}+O\left(z^{2-2 r}\right) .
\end{aligned}
$$

It follows from the Prime Number Theorem and partial summation that

$$
-\log \left(1-C_{r}(q)\right) \sim(r-1) \log z \sim(r-1) \log \log q .
$$

It follows from Conjecture 1 that for these $q$ 's we have

$$
\frac{n_{r}^{*}(q)}{q \log q / \log \log q}<\frac{2+\varepsilon}{r-1} .
$$

Note that Theorem 1 shows that the quantity on the left of $(3)$ is at least $(1-\varepsilon) / r$ for all large $q$, so Theorem 1 may be close to best possible for values of $q$ with many small prime factors. On the other hand, if $q$ has no small prime factors, then Conjecture 1 suggests that $n_{r}^{*}(q)$ is the same order of magnitude as $q \log q$.

Note that $D_{r}(q)$ is smallest when $q$ has the form $q=\prod_{p \leqslant z} p^{r-1}$. When $z$ tends to infinity we have

$$
D_{r}(q)=\prod_{p>z}\left(1-p^{r}\right) \prod_{p \leqslant z}\left(1-p^{-1}\right) \sim e^{-\gamma} / \log \log q .
$$


Conjecture 2 then implies that

$$
n_{r}(q)<\left(2 e^{\gamma}+\varepsilon\right) q \log q \log \log q
$$

for all sufficiently large $q$. This suggests that there may be very little room for improvement in Theorem 4.

The heuristic arguments for Conjectures 1 and 2 are probabalistic in nature, and are similar to that used by Wagstaff [11] for primes in arithmetic progessions. The author wishes to thank D. R. Heath-Brown for correcting an error in the author's original heuristic argument, and also the anonymous referee for suggesting the use of the Borel-Cantelli Lemma and greatly strengthening the conjectures.

Assume first that $(a, q)=1$. The probability that a randomly selected integer in the arithmetic progession $a$ modulo $q$ will be $r$-free is $C_{r}(q)$ (see Cohen and Robinson [1]). Hence the probability that none of the numbers $a, a+q, \ldots, a+$ $(w-1) q$ is $r$-free is $\left(1-C_{r}(q)\right)^{w}$. Assuming independence among the residue classes, the probability that every residue class $a$ modulo $q$ with $(a, q)=1$ contains an $r$-free number among the first $w$ positive integers in the class is

$$
P_{q}=\left(1-\left(1-C_{r}(q)\right)^{w}\right)^{\varphi(q)} \text {. }
$$

If $w=z \log q /\left(-\log \left(1-C_{r}(q)\right)\right)$, then for fixed $z$ we have

$$
\lim _{q \rightarrow \infty} P_{q}= \begin{cases}0 & \text { if } z<1 \\ 1 & \text { if } z>1\end{cases}
$$

This is the argument that leads to the second part of Conjecture 1.

If $A_{q}$ is the event that $n_{r}^{*}(q)<w q$, where $w$ is defined above, then $P\left(A_{q}\right)=1-$ $P_{q}$. If $z>1$, then

$$
\begin{aligned}
P\left(A_{q}\right) & =1-\exp \left(\varphi(q) \log \left(1-\varphi(q)^{-z}\right)\right) \\
& =1-\exp \left(-\varphi(q)^{1-z}+O\left(\varphi(q)^{1-2 z}\right)\right) \\
& =\varphi(q)^{1-z}+O\left(\varphi(q)^{2-2 z}\right) .
\end{aligned}
$$

If $z>2$, then $\sum_{q=1}^{\infty} P\left(A_{q}\right)<\infty$, and the Borel-Cantelli Lemma suggests that $A_{q}$ occurs only finitely many times. If $1<z<2$, then $\sum_{q=1}^{\infty} P\left(A_{q}\right)=\infty$, and we expect that $A_{q}$ occurs infinitely often. This leads to the first part of Conjecture 1.

The argument for Conjecture 2 is similar. If $(a, q)$ is $r$-free, then by a result of Cohen and Robinson [1] the probability that a random integer in the residue class $a$ modulo $q$ will be $r$-free is

$$
D_{r}(a, q)=\prod_{\substack{p \\\left(p^{r}, q\right) \mid a}}\left(1-\left(p^{r}, q\right) p^{-r}\right) .
$$

Hence the probability that every residue class $a$ modulo $q$ with $(a, q) r$-free contains an $r$-free number among the first $w$ positive integers in the class is

$$
P_{q}=\prod_{\substack{a=1 \\(a, q) r \text {-free }}}^{q}\left(1-\left(1-D_{r}(a, q)\right)^{w}\right)
$$


Note that $D_{r}(a, q) \geqslant D_{r}(q)$, so that

$$
P_{q} \geqslant\left(1-\left(1-D_{r}(q)\right)^{w}\right)^{q}
$$

If $w=z \log q /\left(-\log \left(1-D_{r}(q)\right)\right)$ with $z>2$ fixed, it follows that $\sum_{q=1}^{\infty}\left(1-P_{q}\right)<\infty$. This leads to Conjecture 2.

Let $p(a, q)$ be the least prime exceeding $a$ in the arithmetic progression $a$ modulo $q$, and let $P(q)=\max _{(a, q)=1} p(a, q)$. It is interesting to note that when the heuristic argument for the first part of Conjecture 1 is adapted to the case of primes in arithmetic progressions, we arrive at the conjecture that

$$
\varlimsup_{q \rightarrow \infty} \frac{P(q)}{\varphi(q) \log ^{2} q}=2 .
$$

This appears to be in agreement with the numerical data computed by Wagstaff [11]. Wagstaff conjectured that the ratio $P(q) /\left(\varphi(q) \log ^{2} q\right)$ is usually near 1 , but there were a number of numerical examples where the ratio is closer to 2 .

3. The proof of Theorem 1. Let $g(q)$ denote Jacobsthal's function, i.e. $g(q)$ is the least positive integer such that every interval of $g(q)$ consecutive integers contains an integer relatively prime to $q$. Good upper bounds for $g(q)$ have been proved by Iwaniec [6] using sophisticated sieve methods, but here we shall require only the estimate $g(q) \ll q^{\varepsilon}$, which follows easily from the sieve of Eratosthenes.

The proof of Theorem 1 is based on the Chinese Remainder Theorem and the following lemma.

LEMMA 1. Let $\varepsilon>0$ and $m=1+[(1-\varepsilon) \log q /(r \log \log q)]$. If $m$ is sufficiently large, then there exist primes $q_{1}<\cdots<q_{m}$ not dividing $q$ such that

$$
\left(q_{1} q_{2} \cdots q_{m}\right)^{r}<\frac{q}{g(q)+1} \text {. }
$$

Proof. Since $g(q) \ll q^{\delta}$ for every $\delta>0$, it suffices to prove that there exist primes $q_{1}, \ldots, q_{m}$ not dividing $q$ such that

$$
\left(q_{1} q_{2} \cdots q_{m}\right)^{r}<q^{1-\delta} .
$$

It also suffices to treat the case when $q$ is the product of all primes less than $z$. In this case we have $z \sim \log q$, and we can simply choose $m$ primes between $z$ and $2 z$. This is possible because the number of primes between $z$ and $2 z$ is asymptotically $z / \log z$, which exceeds $m$ for $q$ sufficiently large. Since $\log 2 z<(1+\varepsilon / 2) \log \log q$ and

$$
m r<\left(1-\frac{\varepsilon}{2}\right) \frac{\log q}{\log \log q}
$$

for $m$ sufficiently large, it follows that

$$
\left(q_{1} q_{2} \cdots q_{m}\right)^{r}<(2 z)^{m r}<q^{1-\varepsilon^{2} / 4} .
$$

This completes the proof of the lemma. 
We now complete the proof of Theorem 1. Let $\sigma$ be a permutation of the integers $0,1, \ldots, m-1$ such that

$$
\sigma(i)<q_{i+1}^{r}, \quad i=0,1 \ldots, m-1 .
$$

Define $b_{\sigma}$ as the least positive solution of the system of congruences

$$
b_{\sigma}+\sigma(i) \equiv 0\left(\bmod q_{i+1}^{r}\right), \quad i=0,1, \ldots, m-1 .
$$

Choose $k$ to be an integer with $(q, k)=1$ and

$$
\frac{q\left(b_{\boldsymbol{\sigma}}-1\right)}{\left(q_{1} q_{2} \cdots q_{m}\right)^{r}}<k<\frac{q b_{\boldsymbol{\sigma}}}{\left(q_{1} q_{2} \cdots q_{m}\right)^{r}} .
$$

Now let $a_{\sigma}=q b_{\sigma}-k\left(q_{1} q_{2} \cdots q_{m}\right)^{r}$. Note that $\left(a_{\sigma}, q\right)=1,0<a_{\sigma}<q$, and

$$
a_{\sigma}+\sigma(i) q \equiv 0\left(\bmod q_{i+1}^{r}\right), \quad i=0,1, \ldots, m-1 .
$$

It follows that $n_{r}\left(a_{\sigma}, q\right) \geqslant m q$. It remains to show that each $\sigma$ gives rise to a different $a_{\sigma}$, and that the number of permutations $\sigma$ is at least

$$
\exp \left(\frac{1-\varepsilon}{r}\left(1-\frac{\log r}{\log \log q}\right) \log q\right) \text {. }
$$

Let $\sigma$ and $\delta$ be two permutations satisfying (5), and suppose that $a_{\sigma}=a_{\delta}$. Let $n$ be an integer such that $\sigma(n) \neq \delta(n)$. Since $a_{\sigma}=a_{\delta}$, it follows that $b_{\sigma} \equiv b_{\delta}$ $\left(\bmod q_{n+1}^{r}\right)$. Hence $\sigma(n) \equiv \delta(n)\left(\bmod q_{n+1}^{r}\right)$, but this is a contradiction since $0 \leqslant$ $\sigma(n), \delta(n)<q_{n+1}^{r}$. Therefore the $a_{\sigma}$ 's are distinct.

Let $N$ be the number of permutations satisfying (5). An easy counting argument shows that

$$
N=\prod_{i=0}^{m-1} \min \left\{m-i, q_{i+1}^{r}-i\right\} \geqslant(m-l) !,
$$

where $l$ is the least integer such that $q_{l+1}^{r}>m$. Since $l \leqslant \pi\left(m^{1 / r}\right)<m^{1 / 2}$, Stirling's formula yields

$$
\begin{aligned}
\log N & \sim \log (m !)>\frac{1-2 \varepsilon}{r} \frac{\log q}{\log \log q} \log \left(\frac{\log q}{r \log \log q}\right) \\
& >\frac{1-3 \varepsilon}{r} \log q\left(1-\frac{\log r}{\log \log q}\right)
\end{aligned}
$$

for $q$ sufficiently large. Since $\varepsilon$ is arbitrary, Theorem 1 follows.

4. The proof of Theorem 2. The following lemma is probably due to Erdös, but its proof has apparently never appeared in print.

LEMMA 2. Let $p_{n}$ be the nth prime. If $\varepsilon>0$ and $n$ is sufficiently large, then there exist at least $\zeta(r)(1-\varepsilon) n$ consecutive integers each of which is divisible by at least one of the numbers $p_{1}^{r}, p_{2}^{r}, \ldots, p_{n}^{r}$.

Proof. Let $N=[\zeta(r)(1-\varepsilon) n]$. By the Chinese Remainder Theorem, it suffices to show that there exist residue classes $a_{i}$ modulo $p_{i}^{r}$ such that each of the integers $1,2, \ldots, N$ lies in at least one of the residue classes $a_{i}$ modulo $p_{i}^{r}, i=1,2, \ldots, n$. 
Choose $M=M(\varepsilon)$ such that

$$
\prod_{i=M+1}^{\infty}\left(1-p_{i}^{-2}\right)^{-1}<1+\varepsilon .
$$

It then follows that

$$
\prod_{i=1}^{M}\left(1-p_{i}^{-r}\right)<\frac{1+\varepsilon}{\zeta(r)} .
$$

From the interval $[1, N]$, remove all integers in a residue class $a_{1}$ modulo $p_{1}^{r}$, where $a_{1}$ is chosen so as to remove as many integers as possible. Then choose $a_{2}$ modulo $p_{2}^{r}$ so as to remove as many of the remaining integers as possible, and continue in this way for the first $M$ primes. Since at each stage we remove at least the average number of integers in a residue class, the number $S$ of integers remaining after this process satisfies

$$
S<N \prod_{i=1}^{M}\left(1-p_{i}^{-r}\right)<\left(1-\varepsilon^{2}\right) n .
$$

We can then use one prime for each of the remaining $S$ integers, and in this way we can "sieve out" the entire interval using at most $M+\left(1-\varepsilon^{2}\right) n$ primes. It then suffices to take $n>M \varepsilon^{-2}$.

The proof of Theorem 2 is similar to that of Theorem 1. Let

$$
m=\left[\frac{(1-\varepsilon) \log q}{r \log \log q}\right]
$$

and let $q_{1}, q_{2}, \ldots, q_{m}$ be the first $m$ primes. By the prime number theorem,

$$
\log \left(q_{1} \cdots q_{m}\right)<(1+\varepsilon) m \log m<\frac{1-\varepsilon^{2}}{r} \log q .
$$

Since $g(q) \ll q^{\varepsilon}$, it follows that (4) holds. Let $n=[\zeta(r)(1-\varepsilon) m$ ], and choose $b$ in such a way that each of the integers $b+1, b+2, \ldots, b+n$ is divisible by at least one of $q_{1}^{r}, \ldots, q_{m}^{r}$. Then choose $k$ with $(k, q)=1$ and

$$
\frac{q(b-1)}{\left(q_{1} q_{2} \cdots q_{m}\right)^{r}}<k<\frac{q b}{\left(q_{1} q_{2} \cdots q_{m}\right)^{r}} .
$$

With $a=b q-k\left(q_{1} q_{2} \cdots q_{m}\right)^{r}$, it follows that

$$
n_{r}(a, q) \geqslant n q>(1-3 \varepsilon) \frac{\zeta(r)}{r} q \frac{\log q}{\log \log q} .
$$

5. The proof of Theorem 3. Let $q_{1}, q_{2}, \ldots$ be the primes that do not divide $a$. For $m$ large, choose $q$ such that

$$
\begin{aligned}
& q \equiv 1(\bmod a), \\
& n q \equiv-a\left(\bmod q_{n}^{r}\right), \quad n=1,2, \ldots, m, \\
& a\left(q_{1} q_{2} \cdots q_{m}\right)^{r}<q \leqslant 2 a\left(q_{1} q_{2} \cdots q_{m}\right)^{r} .
\end{aligned}
$$


It then follows that $(a, q)=1, a<q$, and $n_{r}(a, q)>m q$. By the prime number theorem,

$$
\frac{1}{r} \log q \sim \log \left(q_{1} q_{2} \cdots q_{m}\right) \sim m \log m .
$$

Hence for $m$ sufficiently large we have

$$
m>\frac{1-\varepsilon}{r} \frac{\log q}{\log \log q} .
$$

6. The proof of Theorem 4. The idea behind the proof of Theorem 4 is that if $(a, q)$ is $r$-free but divisible by many small primes to the power $r-1$, then a number of the form $a+n q$ is "close" to being divisible by an $r$ th power of a prime.

Let $p_{n}$ be the $n$th prime, and define $Q_{m}=p_{1} p_{2} \cdots p_{m}$. A result of Rankin [10] states that

$$
g\left(Q_{m}\right)>\left(e^{\gamma}-\varepsilon\right) \log Q_{m} \log _{2} Q_{m} \frac{\log _{4} Q_{m}}{\left(\log _{3} Q_{m}\right)^{2}}
$$

if $m$ is sufficiently large. Choose $b$ such that $\left(b, Q_{m}\right)=1$ and each of the integers $b+1, b+2, \ldots, b+g\left(Q_{m}\right)-1$ is divisible by at least one of the first $m$ primes. Determine $q^{\prime}$ by

$$
\begin{gathered}
b q^{\prime} \equiv p_{m+1}^{r} \quad\left(\bmod Q_{m}\right), \quad q^{\prime} \equiv 1 \quad\left(\bmod p_{m+1}\right), \\
p_{m+1}^{r}<q^{\prime} \leqslant p_{m+1}^{r}+p_{m+1} Q_{m} .
\end{gathered}
$$

It follows that

$$
q^{\prime} n+p_{m+1}^{r} \equiv q^{\prime}(n+b) \quad\left(\bmod Q_{m}\right) .
$$

Let $a=p_{m+1}^{r} Q_{m}^{r-1}$ and $q=q^{\prime} Q_{m}^{r-1}$. We now have $(a, q)=Q_{m}^{r-1}$ is $r$-free, $a<q$, and $a+n q$ is not $r$-free for $0 \leqslant n \leqslant g\left(Q_{m}\right)-1$. Hence $n_{r}(a, q) \geqslant g\left(Q_{m}\right) q$. Finally, we have $Q_{m+1}^{r-1}<q<Q_{m+1}^{r}$, so that

$$
\log Q_{m}>\frac{1-\varepsilon}{r} \log q
$$

for $m$ sufficiently large.

\section{REFERENCES}

1. E. Cohen and R. L. Robinson, On the distribution of the $k$-free integers in residue classes, Acta Arith. $8(1962 / 63), 283-293$.

2. P. Erdös, Üher die kleinste quadratfreie Zahl einer arithmetischen Reihe, Monatsh. Math. 64 (1960), 314-316.

3. D. R. Heath-Brown, The least square-free number in an arithmetic progression, J. Reine Angew. Math. 332 (1982), 204-220.

4. C. Hooley, A note on square-free numbers in arithmetic progressions, Bull. London Math. Soc. 7 (1975), 133-138.

5. M. Huxley, The difference between consecutive primes, Analytic Number Theory, Proc. Sympos. Pure Math., vol. 24, Amer. Math. Soc., Providence, R. I., 1973, pp. 141-145. 
6. H. Iwaniec, On the problem of Jacobsthal, Demonstratio Math. 11 (1978), 225-231.

7. C. Pomerance, A note on the least prime in an arithmetic progression, J. Number Theory 12 (1980), $218-223$

8. K. Prachar, Über die kleinste quadratfreie Zahl einer arithmetischen Reihe, Monatsh. Math. 62 (1958), 173-176.

9. __ Über die kleinste Primzahl einer arithmetischen Reihe, J. Reine Angew. Math. 206 (1961), 3-4.

10. R. A. Rankin, The difference between consecutive prime numbers. V, Proc. Edinburgh Math. Soc. (2) $13(1962 / 63), 331-332$.

11. S. S. Wagstaff, Greatest of the least primes in arithmetic progressions having a given modulus, Math. Comp. 33 (1979), 1073-1080.

12. R. Warlimont, On squarefree numbers in arithmetic progressions, Monatsh. Math. 73 (1969), 433-448.

13. . Über die kleinsten quadratfreien Zahlen in arithmetischen Progessionen, J. Reine Angew. Math. 250 (1971), 99-106.

Department of Mathematics, Michigan State University, East Lansing, Michigan 48824

Current address: Department of Mathematics, DRB306, University of Southern California, Los Angeles, California 90089-1113 